\title{
PorA types in Neisseria meningitidis serogroup B isolated in Argentina from 2001 to 2003: implications for the design of an outer membrane protein-based vaccine \\ Correspondence \\ Marta Mollerach \\ mmollera@ffyb.uba.ar \\ Received 13 September 2007 \\ Accepted 6 November 2007 \\ Cecilia Sorhouet Pereira, ${ }^{1,2}$ Mabel Regueira ${ }^{2}$ and Marta Mollerach ${ }^{1}$

\author{
${ }^{1}$ Cátedra de Microbiología, Facultad de Farmacia y Bioquímica, Universidad de Buenos Aires, \\ Junin 956, 1113 Buenos Aires, Argentina \\ ${ }^{2}$ Departamento de Bacteriología, Instituto Nacional de Enfermedades Infecciosas - ANLIS 'Dr \\ Carlos G. Malbrán', Av. Velez Sarsfield 563, 1281 Buenos Aires, Argentina
}

\begin{abstract}
Identification of Neisseria meningitidis PorA types remains important, as the PorA protein is a major immunogenic component of several meningococcal vaccines under development. In this study, 191 N. meningitidis serogroup B isolates collected in Argentina through active laboratory-based surveillance from 2001 to 2003 were serosubtyped. Nucleotide sequences of the porA variable region 1 (VR1) and VR2 regions were determined in 52 non-serosubtypeable isolates. A substantial number of distinct VR types were identified, and a new VR2 variant from the P1.16 family was described. This is the first report describing PorA types in N. meningitidis serogroup $B$ isolates in Argentina. Furthermore, the wide diversity of subtypes detected by serosubtyping and genosubtyping reveals the difficulty in designing a useful outer-membrane vaccine applicable in this country. A possible mechanism responsible for altered PorA expression was analysed in two PorA types.
\end{abstract}

\section{INTRODUCTION}

Neisseria meningitidis is an important cause of morbidity and mortality worldwide. To date, there is no satisfactory childhood vaccine available that can protect against all meningococcal strains (Harrison, 2006; Pollard \& Moxon, 2002). Meningococcal capsular polysaccharides of serogroups A, C, Y and W135 are immunoprotective antigens included in vaccines that have been used extensively for decades. Conjugate vaccine against serogroup $\mathrm{C}$ is now in use in several European countries depending on the epidemiological conditions and the health policy in each country (Harrison, 2006; Pollard \& Moxon, 2002). Recently, a quadrivalent vaccine has been licensed by the US Food and Drug Administration that contains A, C, Y and W135 polysaccharides conjugated to diphtheria toxoid. New recommendations for the use of these vaccines have been published (Bilukha \& Rosenstein, 2005). The major advantages of meningococcal conjugate vaccines are immunogenicity in infants, induction of immunological

Abbreviations: INEI, Instituto Nacional de Enfermedades Infecciosas; FSST, fully serosubtyped; MenB, Neisseria meningitidis serogroup B; NSST, non-serosubtypeable; OMP, outer-membrane protein; PSST, partially serosubtyped; $V R$, variable region.

The GenBank/EMBL/DDBJ accession numbers for the porA sequences of the two non-serosubtypeable Neisseria meningitidis serogroup B isolates are DQ411822 and DQ453920. memory and the booster response. Prevention of serogroup B meningococcal disease represents a particularly difficult challenge as the capsular polysaccharide is unable to stimulate the immune response, probably due to its structural similarity to human neural antigens (Finne et al., 1983). Other alternatives for outer-membrane protein (OMP)-based vaccines against this serogroup have been developed (Cartwright et al., 1999; O'Hallahan et al., 2004). All meningococci present either a class 2 or a class 3 OMP referred to as PorB, and most strains also express a class 1 protein (P1 protein), named PorA. This protein was identified as a major inducer of bactericidal antibodies, but, due to its variability, the development of a PorA vaccine has to consider the prevalence of serosubtypes (Peeters et al., 1996; Rosenqvist et al., 1995; Sacchi et al., 2001). A multivalent vaccine could be required, considering the genetic diversity of isolates causing invasive infection in the endemic situation. In contrast, in prolonged outbreaks - the other main epidemiological pattern associated with serogroup B disease - a single clone is generally involved and OMP-based vaccines have been developed and used to control clonal outbreaks with different levels of success (Harrison, 2006; O'Hallahan et al., 2004; Taha \& Alonso, 2007).

Serosubtypes have been defined by reactivity with a set of mAbs. However, nucleotide sequence analyses of porA genes have established that the panel of serosubtyping 
mAbs is not exhaustive and many PorA variants cannot be detected. A two-dimensional structural model containing eight exposed surface loops (loops I-VIII) has been predicted for PorA (van der Ley et al., 1991). Most variability between PorA alleles resides in the variable regions, VR1 and VR2, which correspond to loops I and IV, respectively, in this structure (Maiden et al., 1991; van der Ley et al., 1991). A three-dimensional structural model for Neisseria PorA has recently been predicted, providing information on the spatial relationships of the VRs in the PorA trimer (Derrick et al., 1999). Serosubtype-defining mAbs react with peptide epitopes located in these loops. A fully serosubtyped (FSST) strain has both VR epitopes characterized by mAbs, whereas a partially serosubtyped (PSST) strain has only one VR (VR1 or VR2) characterized by mAbs; neither VR is characterized in non-serosubtypeable (NSST) isolates. Only porA sequencing allows the deduction of VR1 and VR2 sequences from the DNA sequence, but it does not provide information on PorA production at the bacterial surface.

In Argentina, the annual incidence rate of meningococcal disease has been estimated to be around 0.6-2.9 cases per 100000 inhabitants over the last 14 years. In the mid-1990s, there was an increase in the incidence of serogroup C, but serogroup B has been isolated more frequently since 2001 and now represents $60 \%$ of all isolates. Our previous study of 2244 invasive isolates received by the National Reference Laboratory, Instituto Nacional de Enfermedades Infecciosas (INEI) - ANLIS 'Dr Carlos G Malbrán', between 1993 and 2005 showed a high prevalence of NSST isolates (Chiavetta $e t$ al., 2007). In this study, N. meningitidis serogroup B (MenB) isolates collected in the period 2001-2003 through laboratory-based surveillance were studied by serosubtyping, and porA sequencing was performed for NSST isolates. This information may be used to define the most appropriate combination of PorA types that could be included in a hypothetical PorA-based vaccine to be used in Argentina.

\section{METHODS}

Active surveillance. Isolates analysed in this study were recovered from different laboratories in the country during the period 20012003 and were received by the National Reference Laboratory at the INEI - ANLIS 'Dr Carlos G. Malbrán' for active laboratory-based surveillance of invasive disease caused by $N$. meningitidis. Participating laboratories were geographically dispersed among the 23 provinces of the country. Meningococcal disease is an obligatory declared disease. Between 1 January 2001 and 31 December 2003, a total of 1260 cases of meningococcal disease were reported to the Public Health Ministry in the country, giving a mean annual incidence rate of 1.2 per 100000 inhabitants. Reporting criteria included probable and confirmed cases. A confirmed case of meningococcal disease was defined as the isolation of $N$. meningitidis from a normally sterile site. From a total of 347 N. meningitidis isolates received by the National Reference Laboratory at the INEI ANLIS 'Dr Carlos G. Malbrán', 191 were MenB isolates.

Serotyping. All isolates were serosubtyped by whole-cell ELISA (Abdillahi \& Poolman, 1987) against a battery of 14 serosubtype-specific
mAbs provided by the Adolfo Lutz Institute and the National Institute for Public Health and Environment Protection, Bilthoven, The Netherlands (Table 1). Eighty of the $191 \mathrm{MenB}$ isolates (42\%) were NSST, 60 of which were available for analysis by PorA VR genotyping.

Growth of meningococci and DNA preparation. Isolates were propagated on heated blood agar plates in an atmosphere of $5 \% \mathrm{CO}_{2}$ for $18 \mathrm{~h}$. Boiled meningococcal suspensions were used as templates to amplify the porA gene.

PCR amplification of the porA gene. A porA gene product of 1236 bp that included the start codon was obtained by PCR with primers P14 (Sacchi et al., 1998) and P22 (Maiden et al., 1991). PCR conditions were as described previously (Sacchi et al., 1998).

PorA VR genotyping. For PorA VR genotyping, a region of the porA gene that encodes the defined variable epitopes VR1 and VR2 was sequenced using primers U86 and R773 (Sacchi et al., 1998). VR identification was achieved by querying the PorA VR sequence database located at http://neisseria.org/nm/typing/pora (Russell et al., 2004). Full-length sequencing of the porA gene was performed when a new VR variant was detected.

New allelic variants of the porA gene obtained during the study were submitted to GenBank and the PorA VR sequence database.

Sequencing of the porA promoter region. The porA promoter region was amplified and sequenced using primers PorA5 and P1-1 as described previously (Feavers \& Maiden, 1998; van der Ende et al., 1999).

OMP analysis by SDS-PAGE. Preparation of the OMP fraction was performed as described previously (Mocca \& Frasch, 1982) and analysed by SDS-PAGE (Laemmli, 1970). The amount of protein obtained was determined using the Bradford protein assay. Two micrograms of each isolate was analysed by $12 \%$ SDS-PAGE. Proteins in the gels were stained with $0.25 \%$ Coomassie Brilliant Blue. Prestained molecular mass standards were used.

Table 1. Serosubtype-defining mAbs and their identification and epitope locations

\begin{tabular}{|c|c|c|c|c|}
\hline \multirow[t]{2}{*}{ Serosubtype } & \multicolumn{2}{|c|}{$\begin{array}{l}\text { PorA serosubtype } \\
\text { epitope location }\end{array}$} & \multicolumn{2}{|l|}{$\mathrm{mAb}$} \\
\hline & VR1 & VR2 & Identification & Source \\
\hline P1.1 & & + & MN14C2.3 & NIPH \\
\hline P1.2 & & + & MN16C13F4 & NIPH \\
\hline P1.4 & & + & MN20B9.34 & NIPH \\
\hline P1.5 & + & & MN22A9.19 & NIPH \\
\hline P1.6 & & + & MN19D6.13 & $\mathrm{NIPH}$ \\
\hline P1.7 & + & & MN14C11.6 & NIPH \\
\hline P1.9 & & + & MN5A10F & $\mathrm{NIPH}$ \\
\hline $\mathrm{P} 1.10$ & & + & MN20F4.17 & $\mathrm{NIPH}$ \\
\hline $\mathrm{P} 1.12$ & + & & MN20A.10 & $\mathrm{NIPH}$ \\
\hline $\mathrm{P} 1.13$ & & + & MN24H10.75 & $\mathrm{NIPH}$ \\
\hline $\mathrm{P} 1.14$ & & + & MN21G3.17 & $\mathrm{NIPH}$ \\
\hline $\mathrm{P} 1.15$ & & + & MN3C5C & NIPH \\
\hline P1.16 & & + & MN5C11G & $\mathrm{NIPH}$ \\
\hline P1.22-1 & + & & F4-1F1/1G11 & ALI \\
\hline
\end{tabular}

${ }^{*}$ ALI, Adolfo Lutz Institute; NIPH, National Institute for Public Health and Environment Protection, Bilthoven, The Netherlands. 
Western blotting. PorA was identified by Western blotting using PorA-specific mAbs. Proteins were transferred from unstained gels to nitrocellulose membranes using a Bio-Rad Mini Trans-Blot Electrophoretic Transfer Cell according to the manufacturer's instructions. Membranes were blocked with $2 \%$ skimmed milk in PBS for $1 \mathrm{~h}$ at room temperature, washed three times with $0.1 \%$ Tween 20 in PBS and incubated overnight at room temperature with $20 \mathrm{ml}$ of a $1: 1000$ dilution (determined previously) of the mAb. After washing again, the membranes were incubated with horseradish peroxidase-conjugated goat anti-mouse immunoglobulin (SigmaAldrich), washed and developed with 4-chloro-1-naphthol.

\section{RESULTS AND DISCUSSION}

Over the period 2001-2003, $191 \mathrm{MenB}$ isolates from a total of $347 \mathrm{~N}$. meningitidis isolates were received by the National Reference Laboratory at the INEI - ANLIS 'Dr Carlos G. Malbrán'. Serogroup C represented $28 \%$, serogroup $\mathrm{Y} 10 \%$ and the remaining serogroups were $\mathrm{W} 135$ and $\mathrm{X}$. Isolates were recovered from cerebrospinal fluid (77\%), blood (15.2\%) and other sterile sites (7.8\%). PorA phenotyping by whole-cell ELISA showed that only $18.3 \%$ were FSST, whilst $39.8 \%$ were PSST and $41.9 \%$ were NSST. The serosubtype distribution is presented in Table 2.

PorA genotyping was focused on NSST isolates to determine the prevalence of individual PorA types in this group. Only 60/80 NSST isolates could be recovered. Amplification with primers U86 and R773 (Sacchi et al., 1998) produced the expected product from 52 isolates; the porA gene could not be amplified from the remaining eight isolates. After DNA sequencing of porA, the VR1 and VR2 types of the 52 isolates were identified by querying the PorA variable region database. The obtained PorA type distribution is presented in Table 3. A new PorA VR2 peptide sequence, YYTDTNNNLTLVP, was detected in

Table 2. Serosubtypes of MenB isolates in Argentina (20012003)

\begin{tabular}{|lc|}
\hline Serosubtype & No. of isolates (\%) \\
\hline NSST & $80(41.9)$ \\
P1.14 & $34(17.8)$ \\
P1.7,16 1.7 & $32(16.8)$ \\
P1.16 & $14(7.3)$ \\
P1.15 & $10(5.2)$ \\
P1.12 & $8(4.2)$ \\
P1.7,1 & $5(2.6)$ \\
P1.5,2 & $1(0.5)$ \\
P1.12,13 1.2 & $1(0.5)$ \\
P1.4 & $1(0.5)$ \\
P1.5 & $1(0.5)$ \\
P1.6 & $1(0.5)$ \\
P1.10 & $1(0.5)$ \\
Total & $1(0.5)$ \\
\end{tabular}

Table 3. Distribution of PorA types from VR sequencing among NSST MenB isolates in Argentina (2001-2003)

\begin{tabular}{|lc|}
\hline PorA genotype & No. of isolates $(\%)$ \\
\hline P1.21,16-36 $1.19,15$ & $18(30)$ \\
P1.18-1,34 & $9(15)$ \\
P1.22,14-6 & $6(10)$ \\
P1.19-2,13-1 & $4(6.7)$ \\
P1.22,26 & $3(5)$ \\
P1.7-2,3 & $3(5)$ \\
P1.7,16-77* & $2(3.3)$ \\
P1.18-1† & $2(3.3)$ \\
P1.19-2,13-2 $1.7-4,14-6$ & $1(1.7)$ \\
P1.21,16 & $1(1.7)$ \\
P1.7-2,26 & $1(1.7)$ \\
ND & $1(1.7)$ \\
Total & $1(1.7)$ \\
\end{tabular}

ND, porA gene could not be amplified.

${ }^{\star}$ First description of VR2 16-77.

$\uparrow$ Stop codon in the VR2 sequence.

two isolates. This VR type belongs to family 16 and number 77 was assigned to this variant in the PorA database (http:// neisseria.org/nm/typing/pora).

Low expression of PorA has been associated mainly with nucleotide sequence variation in the promoter region of the porA gene (van der Ende et al., 1995). Eleven isolates categorized as NSST were predicted to present a VR type (P1.7 and P1.15) that should have reacted with the mAbs used in the ELISA serosubtyping (mAb MN14C11.6 and MN3C5C, respectively). The DNA sequence of the porA promoter region of nine of these isolates was determined by PCR-based sequencing. Analysis of the poly $(\mathrm{G})$ stretch in the spacer between the putative -10 and -35 regions of the porA promoter revealed that seven isolates had a poly $(\mathrm{G})$ tract of $11 \mathrm{G}$ residues, one isolate had $12 \mathrm{G}$ residues $(\mathrm{Nm} 1849, \mathrm{P} 1.19,15)$ and the other had $10 \mathrm{G}$ residues (Nm 1905, P1.19,15) (Table 4). All isolates showed a similar profile by SDS-PAGE (not shown), revealing the main detectable proteins described previously (PorA, PorB and RmpM) (Frasch et al., 1985; Sánchez et al., 2005). However, only very weak expression of PorA could be detected by SDS-PAGE and Western blotting using $\mathrm{mAb}$ MN3C5C in Nm 1905 [10 nucleotides in the poly $(\mathrm{G})$ tract]. PorA production was similar in the isolates with 12 and 11 nt in the poly $(\mathrm{G})$ stretch.

Serosubtyping has been extensively used for routine epidemiological studies; however, the high percentage of PSST and NSST isolates indicates the need for genetic procedures to determine PorA antigenic variability. The use of nucleotide sequencing to determine the VR peptide sequence is becoming increasingly important for meningococcal surveillance and vaccine design. Nevertheless, 
Table 4. Length of the porA promoter spacer, homopolymeric G tract, VR type and PorA expression for the PSST isolates

$(+)$, Weak; ND, not determined.

\begin{tabular}{|c|c|c|c|c|c|c|c|}
\hline Isolate & Serosubtype & $\begin{array}{c}\text { PorA } \\
\text { genotype }\end{array}$ & porA promoter region sequence & $\begin{array}{c}\text { porA promoter } \\
\text { spacer (nt) }\end{array}$ & $\begin{array}{l}\operatorname{Poly}(G) \\
\text { tract }(n t)\end{array}$ & $\begin{array}{l}\text { PorA presence } \\
\text { by SDS-PAGE }\end{array}$ & Western blot \\
\hline Nm 1955 & P1.7 & P1.7,16-77 & TTTTGCGGGGGGGGGGGTATAA & 17 & 11 & + & + \\
\hline Nm 1849 & P1.15 & $\mathrm{P} 1.19,15$ & TTTTGCGGGGGGGGGGGGTATAA & 18 & 12 & + & + \\
\hline Nm 1905 & P1.15 & $\mathrm{P} 1.19,15$ & TTTTGCGGGGGGGGGGTATAA & 16 & 10 & $(+)$ & $(+)$ \\
\hline Nm 1925 & P1.15 & $\mathrm{P} 1.19,15$ & TTTTGCGGGGGGGGGGGTATAA & 17 & 11 & $\mathrm{ND}$ & $\mathrm{ND}$ \\
\hline Nm 2087 & $\mathrm{P} 1.15$ & $\mathrm{P} 1.19,15$ & TTTTGCGGGGGGGGGGGTATAA & 17 & 11 & ND & $\mathrm{ND}$ \\
\hline $\mathrm{Nm} 2088$ & P1.15 & $\mathrm{P} 1.19,15$ & TTTTGCGGGGGGGGGGGTATAA & 17 & 11 & ND & ND \\
\hline Nm 2135 & P1.15 & $\mathrm{P} 1.19,15$ & TTTTGCGGGGGGGGGGGTATAA & 17 & 11 & ND & ND \\
\hline
\end{tabular}

information regarding the different types of PorA meningococci circulating in various regions of the world is still scarce.

The results presented here, as well as those from previous studies, show the advantages of genosubtyping over serosubtyping (Clarke et al., 2003; Sacchi et al., 2000, 2001; Vicente et al., 2005), as it achieves complete identification of both VR1 and VR2 types. Although this method has the potential to type $100 \%$ of the isolates, the porA gene could not be amplified in 8/60 NSST isolates using primers P14 and P22. These isolates will be studied using alternative primers and by Southern blotting to investigate possible deletion of the porA gene. Nevertheless, the genotypic characterization is not completely satisfactory for deciding on the introduction of a hypothetical PorA-based vaccine without the corresponding information about PorA expression.

It has been suggested that serosubtyping failure may be due to limited accessibility of the mAbs to the corresponding epitopes because of a large amount of capsular polysaccharide or lipopolysaccharide masking the PorA VR epitopes (Sacchi et al., 2000). This hypothesis could explain the lack of recognition of epitopes by mAbs in 11 strains when studied by ELISA. Alternatively, it may be speculated that the exposure of a certain VR epitope may be substantially modified according to the other VR sequence because of a possible subtle modification in the complete protein folding. Promoter sequence is another factor previously described as being involved in PorA expression and in this sense it influences serosubtyping results. The expression level of PorA varies according to the poly $(G)$ stretch spacing of the -10 and -35 regions in the porA promoter. Optimal expression of porA correlated with an $11 \mathrm{nt} \operatorname{poly}(\mathrm{G})$ stretch, whereas moderate expression occurs with poly $(G)$ stretches that are 1 or 2 nt shorter (van der Ende et al., 1995).

The identification of prevalent subtypes is of high value in evaluating the possibility of using multivalent OMP-based vaccines to control endemic MenB disease (Peeters et al., 1996; van der Ley et al., 1993). The results of this study showed wide diversity among the isolates analysed. The situation in Argentina is different from that described in Brazil, where a MenB vaccine is being studied consisting mainly of OMP-containing vesicles expressing the two or three serosubtypes most frequently recovered in that country (Lemos et al., 2006; Sacchi et al., 2001). In Argentina, vaccination with one of the formulas developed to date would provide little benefit, and because of the high diversity of circulating strains, it would be difficult to design a tailor-made vaccine to control meningococcal disease. A vaccine designed for Argentina with the six most prevalent subtypes could target $61.25 \%$ of the strains, and $68.58 \%$ if it includes eight subtypes.

This study provides valuable information about the PorAtype diversity of Argentinean MenB isolates and the implications for the introduction of a PorA-based vaccine. The isolates analysed represent all isolates submitted to the National Reference Laboratory through active laboratorybased surveillance and are currently the best available resource for obtaining information about MenB strains in Argentina. Ongoing laboratory surveillance programmes for invasive meningococcal disease can be used to monitor the appropriateness of existing vaccine formulations and to provide valuable information on which to base the formulation and application of new vaccines that are currently under development.

\section{ACKNOWLEDGEMENTS}

This research was partly supported by a grant (PICT 12210) from ANPCYT (Agencia Nacional de Promoción Cientifica y Tecnológica) and Fundación Roemmers. We thank Elsa Chávez, Adriana Ruzic and Cristina Correa for their technical assistance.

\section{REFERENCES}

Abdillahi, H. \& Poolman, J. T. (1987). Whole-cell ELISA for typing Neisseria meningitidis with monoclonal antibodies. FEMS Microbiol Lett 48, 367-371.

Bilukha, O. O. \& Rosenstein, N. (2005). Prevention and control of meningococcal disease. Recommendations of the Advisory 
Committee on Immunization Practices (ACIP). MMWR Recomm Rep 54 (RR-7), 1-21.

Cartwright, K., Morris, R., Rumke, H., Fox, A., Borrow, R., Begg, N., Richmond, P. \& Pollman, J. (1999). Immunogenicity in UK infants of a novel meningococcal vesicle vaccine containing multiple class 1 (PorA) outer membrane proteins. Vaccine 17, 2612-2619.

Chiavetta, L., Chávez, E., Ruzic, A., Mollerach, M. \& Regueira, M. (2007). Surveillance of Neisseria meningitidis in Argentina, 19932005: distribution of serogroups, serotypes and serosubtypes isolated from invasive disease. Rev Argent Microbiol 39, 21-27 (in Spanish).

Clarke, S. C., Diggle, M. A., Mölling, P., Unemo, M. \& Olcén, P. (2003). Analysis of PorA variable region 3 in meningococci: implications for vaccine policy? Vaccine 21, 2468-2473.

Derrick, J. P., Urwin, R., Suker, J., Feavers, I. M. \& Maiden, M. C. (1999). Structural and evolutionary inference from molecular variation in Neisseria porins. Infect Immun 67, 2406-2413.

Feavers, I. M. \& Maiden, M. C. (1998). A gonococcal porA pseudogene: implications for understanding the evolution and pathogenicity of Neisseria gonorrhoeae. Mol Microbiol 30, 647-656.

Finne, J., Leinonen, M. \& Mäkelä, P. H. (1983). Antigenic similarities between brain components and bacteria causing meningitis. Implications for vaccine development and pathogenesis. Lancet 2, 355-357.

Frasch, C. E., Zollinger, W. D. \& Poolman, J. T. (1985). Serotype antigens of Neisseria meningitidis and a proposed scheme for designation of serotypes. Rev Infect Dis 7, 504-510.

Harrison, L. H. (2006). Prospects for vaccine prevention of meningococcal infection. Clin Microbiol Rev 19, 142-164.

Laemmli, U. K. (1970). Cleavage of structural proteins during the assembly of the head of bacteriophage T4. Nature 227, 680-685.

Lemos, A. P. S., Brandao, A. P., Gorla, M. C. O., Paiva, M. V., Simonsen, V. \& Melles, C. E. A. (2006). Phenotypic characterization of Neisseria meningitidis strains isolated from invasive disease in Brazil from 1990 to 2001. J Med Microbiol 55, 751-757.

Maiden, M. C., Suker, J., McKenna, A. J., Bygraves, J. A. \& Feavers, I. M. (1991). Comparison of the class 1 outer membrane proteins of eight serological reference strains of Neisseria meningitidis. Mol Microbiol 5, 727-736.

Mocca, L. F. \& Frasch, C. E. (1982). Sodium dodecyl sulfatepolyacrylamide gel typing system for characterization of Neisseria meningitidis isolates. J Clin Microbiol 16, 240-244.

O'Hallahan, J., Lennon, D. \& Oster, P. (2004). The strategy to control New Zealand's epidemic of group B meningococcal disease. Pediatr Infect Dis J 23, S293-S298.

Peeters, C. C., Rümke, H. C., Sundermann, L. C., Rouppe van der Voort, E. M., Meulenbelt, J., Schuller, M., Kuipers, A. J., van der Ley, P. \& Poolman, J. T. (1996). Phase I clinical trial with a hexavalent PorA containing meningococcal outer membrane vesicle vaccine. Vaccine $\mathbf{1 4}$, 1009-1015.
Pollard, A. J. \& Moxon, E. R. (2002). The meningococcus tamed? Arch Dis Child 87, 13-17.

Rosenqvist, E., Hoiby, E. A., Wedege, E., Bryn, K., Kolberg, J., Klem, A., Ronnild, E., Bjune, G. \& Nokleby, H. (1995). Human antibody responses to meningococcal outer membrane antigens after three doses of the Norwegian group B meningococcal vaccine. Infect Immun 63, 4642-4652.

Russell, J. E., Jolley, K. A., Feavers, I. M., Maiden, M. C. \& Sukert, J. (2004). PorA variable regions of Neisseria meningitidis. Emerg Infect Dis 10, 674-678.

Sacchi, C. T., Lemos, A. P., Brandt, M. E., Withey, A. M., Melles, C. E. A., Solari, C. A., Frasch, C. E. \& Mayer, L. W. (1998). Proposed standardization of Neisseria meningitidis PorA variable-region typing nomenclature. Clin Diagn Lab Immunol 5, 845-855.

Sacchi, C. T., Withey, A. M., Popovic, T., Beall, D. S., Reeves, M. W., Plikaytis, B. D., Rosenstein, N. E., Perkins, B. A., Tondella, M. L. \& Mayer, L. W. (2000). Diversity and prevalence of PorA types in Neisseria meningitidis serogroup B in the United States, 1992-1998. J Infect Dis 182, 1169-1176.

Sacchi, C. T., Lemos, A. P., Popovic, T., de Morais, J. C., Whitney, A. M., Melles, C. E. A., Brondi, L. M. G., Monteiro, L. M. C., Paiva, M. V. \& other authors (2001). Serosubtypes and PorA types of Neisseria meningitidis serogroup B isolated in Brazil during 1997-1998. Overview and implications for vaccine development. J Clin Microbiol 39, 2897-2903.

Sánchez, S., Abel, A., Arenas, J., Criado, M. T. \& Ferreirós, C. M. (2005). Cross-linking analysis of antigenic outer membrane protein complex of Neisseria meningitidis. Res Microbiol 157, 136-142.

Taha, M. K. \& Alonso, J. M. (2007). Meningococcal vaccines: to eradicate the disease, not the bacterium. Hum Vaccin 3, 149-152.

van der Ende, A., Hopman, C. T., Zaat, S., Essink, B. B., Berkhout, B. \& Dankert, J. (1995). Variable expression of class 1 outer membrane protein in Neisseria meningitidis is caused by variation in the spacing between -10 and -35 regions of the promoter. J Bacteriol 177, $2475-2480$.

van der Ende, A., Hopman, C. T. P. \& Dankert, J. (1999). Deletion of porA by recombination between clusters of repetitive extragenic palindromic sequences in Neisseria meningitidis. Infect Immun 67, 2928-2934.

van der Ley, P., Heckles, J. E., Virji, M., Hoogerhout, P. \& Poolman, J. T. (1991). Topology of outer membrane porins in pathogenic Neisseria spp. Infect Immun 59, 2963-2971.

van der Ley, P., van der Biezen, J., Hohenstein, P., Peeters, C. \& Poolman, J. T. (1993). Use of transformation to construct antigenic hybrids of the class 1 outer membrane protein in Neisseria meningitidis. Infect Immun 61, 4217-4224.

Vicente, D., Esnal, O., Michaus, L., López de Goicoechea, M. J., Cisterna, R. \& Pérez-Trallero, E. (2005). Prevalence of genosubtypes (PorA types) of serogroup B invasive meningococcus in the north of Spain from 2000 to 2003. J Med Microbiol 54, 381-384. 